\title{
Dehn twists have roots
}

\author{
DAN MARGALIT \\ SAUL SCHLEIMER
}

\begin{abstract}
We show that every Dehn twist in the mapping class group of a closed, connected, orientable surface of genus at least two has a nontrivial root.
\end{abstract}

$20 \mathrm{~F} 28$

Let $S_{g}$ denote a closed, connected, orientable surface of genus $g$. We denote by $\operatorname{Mod}\left(S_{g}\right)$ its mapping class group: the group of homotopy classes of orientation preserving homeomorphisms of $S_{g}$. In this note, we demonstrate:

Fact If $g \geq 2$, then every Dehn twist in $\operatorname{Mod}\left(S_{g}\right)$ has a nontrivial root.

It follows from the classification of elements in $\operatorname{Mod}\left(S_{1}\right) \cong \operatorname{SL}(2, \mathbb{Z})$ that Dehn twists are primitive in the mapping class group of the torus.

For Dehn twists about separating curves, the fact is well-known: if $c$ is a separating curve then a square root of the left Dehn twist $T_{c}$ is obtained by twisting one side of $c$ through an angle of $\pi$. We construct roots of Dehn twists about nonseparating curves in two ways.

Geometric construction Fix $g \geq 2$. Let $P$ be a regular $(4 g-2)$-gon. Glue opposite sides to obtain a surface $Q \cong S_{g-1}$. The rotation of $P$ about its center through angle $2 \pi k /(2 g-1)$ induces a periodic map $f_{k}$ of $Q$. Notice that $f_{k}$ fixes the points $x, y \in Q$ that are the images of the vertices of $P$ and induces a rotation through angle $-4 \pi k /(2 g-1)$ about each. Let $R$ be the surface obtained from $Q$ by removing small open disks centered at $x$ and $y$. Define $f=f_{g^{2}}^{-1} \mid R$.

Modify $f$ by an isotopy supported in a collar of $\partial R$ so that $f \mid \partial R$ is the identity and $f$ restricts to a $(g-1) /(2 g-1)$-right Dehn twist in each annulus. Identify the components of $\partial R$ to obtain a surface $S \cong S_{g}$. The image of $\partial R$ in $S$ is a nonseparating curve; call it $d$. We see that $\left(f T_{d}\right)^{2 g-1}=T_{d}$, as desired. 
Algebraic construction Let $c_{1}, \ldots, c_{k}$ be curves in $S_{g}$ where $c_{i}$ intersects $c_{i+1}$ once for each $i$, and all other pairs of curves are disjoint. If $k$ is odd, then a regular neighborhood of $\bigcup c_{i}$ has two boundary components, say $d_{1}$ and $d_{2}$, and we have a relation in $\operatorname{Mod}\left(S_{g}\right)$ :

$$
\left(T_{c_{1}}^{2} T_{c_{2}} \cdots T_{c_{k}}\right)^{k}=T_{d_{1}} T_{d_{2}} .
$$

This relation comes from the Artin group of type $B_{n}$, in particular, the factorization of the central element in terms of standard generators. The relation also follows from the $D_{2 p}$ case of [2, Proposition 2.12(i)]. If $k=2 g-1$ the curves $d_{1}$ and $d_{2}$ are isotopic nonseparating curves; call this isotopy class $d$. Using the fact that $T_{d}$ commutes with each $T_{c_{i}}$, we see that

$$
\left[\left(T_{c_{1}}^{2} T_{c_{2}} \cdots T_{c_{2 g-1}}\right)^{1-g} T_{d}\right]^{2 g-1}=T_{d} .
$$

In the remainder of the paper, we find roots for several analogues of Dehn twists.

Roots of half-twists We denote by $S_{0, n}$ a two-sphere with $n$ punctures (or cone points). Let $d$ be a curve in $S_{0,2 g+2}$ with two punctures on one side and $2 g$ on the other. On the side of $d$ with two punctures we perform a left half-twist. On the other side of $d$ we perform a $(g-1) /(2 g-1)$-right Dehn twist by arranging the punctures so that one puncture is in the middle and the other punctures rotate around this central puncture. The $(2 g-1)-$ st power of the composition is a left half-twist about $d$. Thus, we have roots of half-twists in $\operatorname{Mod}\left(S_{0,2 g+2}\right)$ for $g \geq 2$. Forgetting the central puncture gives roots of half-twists in $\operatorname{Mod}\left(S_{0,2 g+1}\right)$.

In the geometric construction, reflection through the center of the polygon $P$ induces a hyperelliptic involution of the surface $S$. In the algebraic construction there is a hyperelliptic involution preserving each curve $c_{i}$. In either case there is an induced orbifold double covering $S_{g} \rightarrow S_{0,2 g+2}$ and the root of the Dehn twist descends to the given root of the half-twist in $\operatorname{Mod}\left(S_{0,2 g+2}\right)$ [1, Theorem 1 plus Corollary 7.1].

Roots of elementary matrices If we consider the $\operatorname{map} \operatorname{Mod}\left(S_{g}\right) \rightarrow \operatorname{Sp}(2 g, \mathbb{Z})$ given by the action of $\operatorname{Mod}\left(S_{g}\right)$ on $H_{1}\left(S_{g}, \mathbb{Z}\right)$ we also see that elementary matrices have roots in $\operatorname{Sp}(2 g, \mathbb{Z})$ :

$$
\left(\begin{array}{rrrr}
1 & 0 & 0 & 1 \\
0 & 1 & 0 & 0 \\
0 & 1 & -1 & 1 \\
0 & 1 & -1 & 0
\end{array}\right)^{3}=\left(\begin{array}{llll}
1 & 1 & 0 & 0 \\
0 & 1 & 0 & 0 \\
0 & 0 & 1 & 0 \\
0 & 0 & 0 & 1
\end{array}\right)
$$

By stabilizing we obtain cube roots of elementary matrices in $\operatorname{Sp}(2 g, \mathbb{Z})$ for $g \geq 2$. 
Roots of Nielsen transformations Let $F_{n}$ denote the free group generated by elements $x_{1}, \ldots, x_{n}$. Let $\operatorname{Aut}\left(F_{n}\right)$ denote the group of automorphisms of $F_{n}$, and assume $n \geq 2$. A Nielsen transformation is an element of $\operatorname{Aut}\left(F_{n}\right)$ conjugate to the one given by $x_{1} \mapsto x_{1} x_{2}$ and $x_{k} \mapsto x_{k}$ for $2 \leq k \leq n$. The following automorphism is the square root of a Nielsen transformation in $\operatorname{Aut}\left(F_{n}\right)$ for $n \geq 3$ :

$$
\begin{aligned}
& x_{1} \mapsto x_{1} x_{3} \\
& x_{2} \mapsto x_{3}^{-1} x_{2} x_{3} \\
& x_{3} \mapsto x_{3}^{-1} x_{2}
\end{aligned}
$$

Passing to quotients, this gives a square root of a Nielsen transformation in $\operatorname{Out}\left(F_{n}\right)$ and, multiplying by $-\mathrm{Id}$, a square root of an elementary matrix in $\operatorname{SL}(n, \mathbb{Z}), n \geq 3$. Also, our roots of Dehn twists in $\operatorname{Mod}\left(S_{g}\right)$ can be modified to work for once-punctured surfaces, thus giving "geometric" roots of Nielsen transformations in $\operatorname{Out}\left(F_{n}\right)$.

Other roots If $f \in \operatorname{Mod}\left(S_{g}\right)$ is a root of a Dehn twist $T_{d}$, then $f$ commutes with $T_{d}$. Since $f T_{c} f^{-1}=T_{f(c)}$ for any curve $c$, we see that $f$ fixes $d$. In the complement of $d$, the class $f$ must be periodic. This line of reasoning translates to $\operatorname{GL}(n, \mathbb{Z})$ and $\operatorname{Aut}\left(F_{n}\right)$ : roots correspond to torsion elements in $\operatorname{GL}(n-1, \mathbb{Z})$ and $\operatorname{Aut}\left(F_{n-1}\right)$, respectively. In all cases, one can show that the degree of the root is equal to the order of the torsion element.

Acknowledgments We thank Serge Cantat for a useful conversation. We are grateful to W B R Lickorish for pointing out a mistake in an earlier draft.

\section{References}

[1] J S Birman, H M Hilden, On the mapping class groups of closed surfaces as covering spaces, from: "Advances in the theory of Riemann surfaces (Proc. Conf., Stony Brook, N.Y., 1969)", (L V Ahlfors et al, editors), Ann. of Math. Studies 66, Princeton Univ. Press (1971) 81-115 MR0292082

[2] C Labruère, L Paris, Presentations for the punctured mapping class groups in terms of Artin groups, Algebr. Geom. Topol. 1 (2001) 73-114 MR1805936

Department of Mathematics, 503 Boston Ave, Tufts University

Medford, MA 02155, USA

Department of Mathematics, University of Warwick

Coventry, CV4 7AL, UK

dan.margalit@tufts.edu, s.schleimer@warwick.ac.uk

Proposed: Walter Neumann

Seconded: Joan Birman, Rob Kirby
Received: 31 October 2008

Revised: 30 December 2008 